\title{
Search and analysis of blue straggler stars in open clusters ${ }^{\star}$
}

\author{
F. De Marchi ${ }^{1}$, F. De Angeli ${ }^{2}$, G. Piotto ${ }^{1}$, G. Carraro ${ }^{1,3,4}$, and M. B. Davies ${ }^{5}$ \\ 1 Dipartimento di Astronomia, Università di Padova, vicolo dell'Osservatorio 2, Padova 35122, Italy \\ e-mail: demarchi-piotto@pd.astro.it \\ 2 Institue of Astronomy, University of Cambridge, Madingley Road, Cambridge CB3 OHA, UK \\ e-mail: fda@ast.cam.ac.uk \\ 3 Andes Fellow, Departamento de Astronómia, Universidad de Cile, Casilla 36-D, Santiago, Chile \\ 4 Astronomy Department, Yale University, PO Box 208101 New Haven, CT 06520-8101, USA \\ e-mail: gcarraro@das.uchile.cl \\ 5 Lund Observatory, Box 43, 22100 Lund, Sweden \\ e-mail: mbd@astro.lu.se
}

Received 23 January 2006 / Accepted 14 July 2006

ABSTRACT

\begin{abstract}
Aims. This paper presents a new homogeneous catalogue of blue straggler stars (BSS) in Galactic open clusters. Methods. Photometric data for 216 clusters were collected from the literature and 2782 BSS candidates were extracted from 76 of them.

Results. We found that the anticorrelation of BSS frequency vs. total magnitude identified in similar studies conducted on Galactic globular clusters extends to the open cluster regime: clusters with smaller total magnitude tend to have higher BSS frequencies. Moreover, a clear correlation between the BSS frequency (obtained normalising the total number of BSS either to the total cluster mass or, for the older clusters, to the total number of clump stars) and the age of the clusters was found. A simple model is developed here to try to explain this last and new result. The model allows us to ascertain the important effect played by mass loss in the evolution of open clusters.
\end{abstract}

Key words. catalogs - stars: blue stragglers - Galaxy: globular clusters: general - Galaxy: open clusters and associations: general stars: Hertzsprung-Russell (HR) and C-M diagrams

\section{Introduction}

Blue straggler stars (BSS) occupy a region above the turn-off (TO) point in the colour-magnitude diagram (CMD) of star clusters, where no stars are expected on the basis of standard stellar evolution, if we assume that all cluster stars are coeval. A number of exotic explanations have been invoked to interpret the nature of these stars. Briefly, it has been suggested that these stars are the outcome of stellar collisions (Benz \& Hills 1987; Lombardi et al. 1996) or of mass exchange in close binary systems (McCrea 1964; Eggen \& Iben 1989; Mateo et al. 1990). Other possible scenarios include second-generation stars (Eggen \& Iben 1988), accretion of gas from the interstellar surrounding medium (Williams 1964), and capture of field stars by a star cluster.

Interestingly, BSS are ubiquitous, as they have been found in globular clusters (GCs), dwarf galaxies, open clusters (OCs), and in the Galactic field. Therefore, important constraints on their nature and possible formation mechanisms can be derived from a comparative study of the BSS properties in different environments. In the past two decades, a huge amount of new, high-quality CMDs from CCD photometry from groundbased and space facilities have become available. Recently,

* Tables 2 and 3 are only available in electronic form at the CDS via anonymous ftp to cdsarc.u-strasbg.fr (130.79.128.5) or via http://cdsweb.u-strasbg.fr/cgi-bin/qcat?]/A+A/459/489
Piotto et al. (2004, hereafter PDK04) published a new photometrically homogeneous catalogue of BSS extracted from the CMDs from HST observations of more than $1 / 3$ of the known Galactic GCs (Piotto et al. 2002). The present paper aims to complement the PDK04 catalogue by extending the study of BSS properties to the different environment that is typical of OCs.

The catalogue presented in this paper can be considered as an important extension of the effort made by Ahumada \& Lapasset (1995, hereafter AL95) to compile a catalogue of 969 BSS in 390 OCs, based on the photometry obtained with photographic plates and photoelectric detectors in the $60 \mathrm{~s}, 70 \mathrm{~s}$, and 80s. The main conclusions drawn from their catalogue are that (1) BSS are present in clusters of all ages; (2) the fraction of BSS increases with the richness and age of a cluster; and (3) BSS show a remarkable degree of central concentration. Moreover, the number of BSS over the number of main sequence stars seems constant up to an age of $400 \mathrm{Myr}$.

The main reasons that led us to perform a new search of BSS in OCs are the following: (1) the AL95 catalogue was based on a highly inhomogeneous set of data; (2) a large number of new, high-quality photometric studies of OC stars have appeared since AL95; and (3) the BSS selection criteria needed in our opinion to be revised, especially for young OCs. Most important, in order to estimate the relative number of BSS in different clusters, the total number of BSS must be normalised to some reference stellar population in the same cluster. The choice made 
by AL95 to normalise the BSS counts to the main sequence stars creates some problems, especially in young clusters, where it is difficult to decide whether a star is a main sequence, a blue straggler, or a field star.

The layout of this paper is as follows. In Sect. 2 we present the new database. In Sect. 3 we describe a few interesting properties highlighted by the new catalogue. In Sect. 4 we develop a simple model in order to interpret the observed BSS frequency in OCs. Section 5 presents our conclusions.

\section{The new database}

We collected a database of $B, V$ (in some cases $I$ ) band CCD photometry of 216 galactic OCs from the literature. The clusters in this new catalogue have ages ranging from 5.5 to $10^{4}$ Myr. From the entire sample we extracted a new catalogue of 2782 BSS candidates. This new catalogue can be considered an updated version of the AL95 catalogue. Recent CCD photometry was collected for 136 clusters out of the original 390 in the AL95 catalogue. Moreover, the database was extended with 80 additional OCs for which CCD photometry is now available, but which were not included in the AL95 catalogue.

Out of the whole sample of 216 open clusters, only 76 (the oldest ones) show BSS candidates. Fifty-nine of these clusters had sufficiently deep photometry to be considered suitable for the kind of analysis we were interested in, which involves estimating the total mass from the integrated magnitude (see Sect. 3.1). Moreover, to avoid contamination effects from field stars, we decided to select for our analysis only those objects with a shorter distance from the cluster centre than the angular radius of the cluster given in the WEBDA archive ${ }^{1}$. After applying this selection, we were left with 774 BSS candidates.

The list of these 59 clusters used in our analysis and their relevant parameters is found in Table 1. Column 2 gives the logarithm of the age, Col. 3 is the distance of the cluster in parsecs derived via isochrone fitting, Cols. 4 and 5 list the integrated magnitudes (the first value is calculated based on the catalogue used for the present work, see Sect. 3.1, the second one from Lata et al. 2002), Col. 6 is the radius in arcmin from the WEBDA archive, Cols. 7 and 8 are the observed numbers of BSS and clump stars inside the cluster radius respectively. Column 9 lists the photometric bands used for this work $(B V$ or $V I$ ).

The entire catalogue (extracted from the oldest 76 clusters and without any selection in radius) will be published in electronic form at the CDS and available at the Padova Globular Cluster Group web pages (http://dipastro.pd.astro.it/ globulars).

Table 2 (available online at the CDS) lists all the 216 clusters analysed to extract the current catalogue. The following parameters are provided for each entry: right ascension and declination, reddening, distance modulus, logarithm of age, apparent cluster radius, number of BSS candidates within the apparent radius and without limitation on the distance from the centre, number of clump stars, and reference to the adopted photometry (all the references are listed in the file refs.dat, also available on-line).

Table 3 (available online at the CDS) lists all the 2782 BSS candidates. Column 1 provides the name of the cluster where the candidate was found. The presence of an asterisk in Col. 2 indicates the existence of a special note referring to this entry (all the notes are in the file notes.dat also available online). Columns 3-6 give the identification number, the distance

\footnotetext{
${ }^{1}$ http://obswww. unige.ch/webda/navigation.html
}

from the centre, the magnitude and the colour respectively for each candidate.

The content of the electronic tables is described in details in the file ReadMe also available on-line.

Although based on a smaller number of OCs than the one by AL95, the new catalogue contains a larger number of BSS and has the advantage of being much more homogeneous and therefore better suited to comparative analyses of BSS in different environments.

\subsection{Selection criterion}

BSS occupy a region of the CMD usually bluer and brighter than the TO. To determine the TO position, we superimposed the Girardi et al. (2000) theoretical isochrones to the observed CMD. The isochrone fitting also provided us with an estimate of the apparent distance modulus and age for each cluster.

To properly account for the photometric errors and the differential reddening, we defined a BSS selection region in the following way:

- we defined a main sequence for binaries $\left(V_{B i},(B-V)_{B i}\right)$ with equal mass components, shifting the theoretical zeroage main sequence (ZAMS) $\left(V_{i},(B-V)_{i}\right) 0.75$ in magnitude $V$ toward brighter magnitudes

$$
\begin{aligned}
& V_{B i}=V_{i}-0.75 \\
& (B-V)_{B i}=(B-V)_{i}
\end{aligned}
$$

- we then defined the two borders ( 1 for left and $r$ for right) of the selection region

$$
\begin{aligned}
& V_{l_{i}}=V_{i}+\Delta V \\
& (B-V)_{1 i}=(B-V)_{i}-\Delta(B-V) \\
& V_{\mathrm{r} i}=V_{B i}-\Delta V \\
& (B-V)_{\mathrm{r} i}=(B-V)_{B i}+\Delta(B-V)
\end{aligned}
$$

where $\Delta V$ and $\Delta(B-V)$ were set to 0.15 mag to take photometric errors and differential reddening into account.

Figure 1 shows an example of the selection region on the CMD of NGC 7789. The same procedure has been applied to the CMDs in $(V, V-I)$.

\section{Analysis}

This section presents our analysis of the selected BSS catalogue. The estimate of the main parameters used in this study is explained in details and the most relevant correlations among the observed quantities are described.

\subsection{Integrated magnitudes}

For each cluster we computed the integrated magnitude and the total mass, considering as cluster members only the stars falling inside a certain angular radius $R$ from the centre (the adopted values of $R$ are those listed on WEBDA and in Table 1$)^{2}$.

We calculated the total magnitude $M_{V}^{<5}$ as the sum of the luminosity of all the stars with absolute magnitude $M_{V}<5$ and

\footnotetext{
2 Note that the lack of unresolved stars in our total magnitude estimate is partly compensated for by the likely presence of some field stars left in the bright star catalogue.
} 
Table 1. List of open clusters and relevant parameters.

\begin{tabular}{|c|c|c|c|c|c|c|c|c|}
\hline ID & $\log t$ & Dist. (pc) & $M_{V}^{<5}$ & $M_{V}^{\mathrm{LP} 02}$ & $R$ & $N_{\text {BSS }}$ & $N_{\mathrm{cl}}$ & Band \\
\hline ArpMadore 2 & 9.40 & 13320 & -3.48 & & 1.00 & 2 & 3 & $B V$ \\
\hline Berkeley 10 & 8.80 & 2294 & -5.21 & . & 5.00 & 7 & & $B V$ \\
\hline Berkeley 12 & 9.50 & 3289 & -2.67 & & 2.00 & 14 & & $B V$ \\
\hline Berkeley 14 & 9.20 & 5495 & -6.54 & -4.07 & 2.50 & 40 & 5 & $B V$ \\
\hline Berkeley 20 & 9.78 & 8387 & -2.06 & $\ldots$ & 1.00 & 4 & $\ldots$ & $B V$ \\
\hline Berkeley 21 & 9.34 & 5012 & -4.91 & -3.41 & 2.50 & 17 & . & $B V$ \\
\hline Berkeley 22 & 9.35 & 6677 & -3.00 & -3.27 & 0.50 & 3 & 2 & $B V$ \\
\hline Berkeley 23 & 8.90 & 6918 & -4.91 & $\ldots$ & 2.00 & 3 & $\ldots$ & $B V$ \\
\hline Berkeley 29 & 9.50 & 15180 & -3.77 & -4.64 & 3.00 & 3 & 2 & $B V$ \\
\hline Berkeley 30 & 8.48 & 4786 & -4.50 & -5.11 & 1.50 & 1 & . & $B V$ \\
\hline Berkeley 31 & 9.31 & 7185 & -3.81 & -2.57 & 2.50 & 13 & 4 & $B V$ \\
\hline Berkeley 32 & 9.53 & 3496 & -4.00 & -2.97 & 3.00 & 19 & 4 & $B V$ \\
\hline Berkeley 33 & 8.70 & 3855 & -4.47 & -4.61 & 2.00 & 1 & & $B V$ \\
\hline Berkeley 39 & 9.78 & 3802 & -3.69 & -4.28 & 3.50 & 18 & 5 & $B V$ \\
\hline Berkeley 81 & 9.00 & 3006 & -4.74 & -4.70 & 2.50 & 9 & $\ldots$ & $B V$ \\
\hline Berkeley 99 & 9.50 & 4943 & -4.36 & -3.66 & 5.00 & 6 & $\ldots$ & $B V$ \\
\hline Collinder 74 & 9.11 & 2514 & -3.85 & -2.88 & 2.50 & 5 & & $B V$ \\
\hline Collinder 110 & 9.11 & 2415 & -5.07 & $\ldots$ & 9.00 & 18 & 7 & $B V$ \\
\hline Collinder 261 & 9.85 & 2518 & -3.92 & -2.87 & 4.50 & 35 & 6 & $B V$ \\
\hline IC 1311 & 9.20 & 6289 & -4.02 & -3.90 & 2.50 & 3 & & $B V$ \\
\hline IC 4651 & 9.05 & 882 & -1.20 & -2.89 & 5.00 & 1 & & $B V$ \\
\hline King 2 & 9.78 & 6003 & -3.41 & $\ldots$ & 2.50 & 20 & 5 & $B V$ \\
\hline King 11 & 9.55 & 2500 & -3.59 & $\ldots$ & 2.50 & 10 & & $B V$ \\
\hline Melotte 66 & 9.60 & 4018 & -6.40 & $\ldots$ & 7.00 & 29 & 9 & $V I$ \\
\hline Melotte 105 & 8.32 & 2210 & -4.85 & -4.27 & 2.50 & 1 & $\ldots$ & $B V$ \\
\hline NGC 188 & 9.63 & 2047 & -3.91 & -2.86 & 8.50 & 30 & & $B V$ \\
\hline NGC 1193 & 9.90 & 5207 & -3.02 & -3.00 & 1.50 & 11 & & $B V$ \\
\hline NGC 1245 & 8.90 & 3012 & -4.50 & -3.60 & 4.50 & 7 & 5 & $B V$ \\
\hline NGC 1907 & 8.57 & 1558 & -4.26 & -6.19 & 3.50 & 2 & $\ldots$ & $B V$ \\
\hline NGC 2112 & 9.30 & 850 & -3.04 & -1.88 & 9.00 & 6 & $\ldots$ & $B V$ \\
\hline NGC 2141 & 9.40 & 3802 & -4.01 & $\ldots$ & 5.00 & 8 & 6 & $B V$ \\
\hline NGC 2158 & 9.30 & 3899 & -5.55 & -2.81 & 2.50 & 28 & 8 & $B V$ \\
\hline NGC 2194 & 8.75 & 2805 & -5.86 & $\ldots$ & 4.50 & 6 & $\ldots$ & $B V$ \\
\hline NGC 2204 & 9.20 & 3981 & -5.47 & -4.65 & 5.00 & 6 & 6 & $B V$ \\
\hline NGC 2243 & 9.45 & 3976 & -3.26 & -2.67 & 2.50 & 7 & 3 & $B V$ \\
\hline NGC 2266 & 9.00 & 3148 & -3.99 & -3.83 & 2.50 & 2 & $\ldots$ & $B V$ \\
\hline NGC 2420 & 9.30 & 2449 & -2.90 & -3.44 & 2.50 & 3 & 1 & $B V$ \\
\hline NGC 2477 & 9.00 & 1259 & -5.11 & -5.70 & 7.50 & 6 & $\ldots$ & $B V$ \\
\hline NGC 2506 & 9.25 & 3089 & -4.51 & -4.31 & 6.00 & 8 & 5 & $B V$ \\
\hline NGC 2627 & 9.15 & 1858 & -3.59 & -4.84 & 4.00 & 7 & 3 & $V I$ \\
\hline NGC 2660 & 9.03 & 2328 & -3.79 & -4.08 & 1.75 & 5 & 6 & $B V$ \\
\hline NGC 2682 & 9.50 & 865 & -2.93 & -3.16 & 12.50 & 14 & 3 & $B V$ \\
\hline NGC 5999 & 8.60 & 2046 & -3.53 & -3.85 & 1.50 & 2 & $\ldots$ & $B V$ \\
\hline NGC 6005 & 8.95 & 2018 & -4.50 & -3.66 & 2.50 & 6 & 6 & $B V$ \\
\hline NGC 6067 & 8.10 & 2241 & -6.66 & -6.18 & 7.00 & 1 & & $B V$ \\
\hline NGC 6253 & 9.48 & 1727 & -3.09 & -2.68 & 2.00 & 10 & 4 & $B V$ \\
\hline NGC 6791 & 9.95 & 3695 & -5.10 & -4.14 & 5.00 & 119 & $\ldots$ & $B V$ \\
\hline NGC 6819 & 9.38 & 2349 & -4.10 & $\ldots$ & 2.50 & 5 & 4 & $B V$ \\
\hline NGC 6939 & 9.06 & 1862 & -4.13 & $\ldots$ & 5.00 & 9 & 7 & $B V$ \\
\hline NGC 7044 & 9.10 & 2989 & -4.59 & -3.93 & 3.00 & 9 & $\ldots$ & $V I$ \\
\hline NGC 7789 & 9.23 & 1888 & -5.19 & $\ldots$ & 12.50 & 17 & & $B V$ \\
\hline Pismis 2 & 9.06 & 3311 & -5.13 & $\ldots$ & 2.00 & 17 & 7 & $B V$ \\
\hline Pismis 3 & 9.03 & 1361 & -4.11 & $\ldots$ & 2.50 & 12 & 6 & $B V$ \\
\hline Pismis 18 & 9.00 & 1941 & -3.89 & $\ldots$ & 2.00 & 2 & 4 & $B V$ \\
\hline Saurer 2 & 9.30 & 6274 & -5.48 & $\cdots$ & 2.00 & 54 & 6 & $V I$ \\
\hline Tombaugh 1 & 9.20 & 3006 & -4.07 & $\ldots$ & 2.50 & 3 & $\ldots$ & $V I$ \\
\hline Tombaugh 2 & 9.20 & 9419 & -5.14 & $\ldots$ & 1.50 & 7 & 6 & $B V$ \\
\hline Tombaugh 5 & 8.30 & 1754 & -6.06 & $\ldots$ & 7.00 & 3 & $\ldots$ & $B V$ \\
\hline Trumpler 5 & 9.50 & 2995 & -5.88 & -5.06 & 7.00 & 60 & 14 & $B V$ \\
\hline
\end{tabular}

angular distance from the centre smaller than $R$. The adopted limiting absolute magnitude is a compromise between the necessity of reaching faint magnitudes, in order to include as many cluster stars as possible, and of having enough clusters with deep enough available photometry to guarantee a homogeneous determination of the total cluster magnitude.

Recently, Lata et al. (2002, hereafter LP02) published a catalogue of integrated magnitudes for a large sample of open 


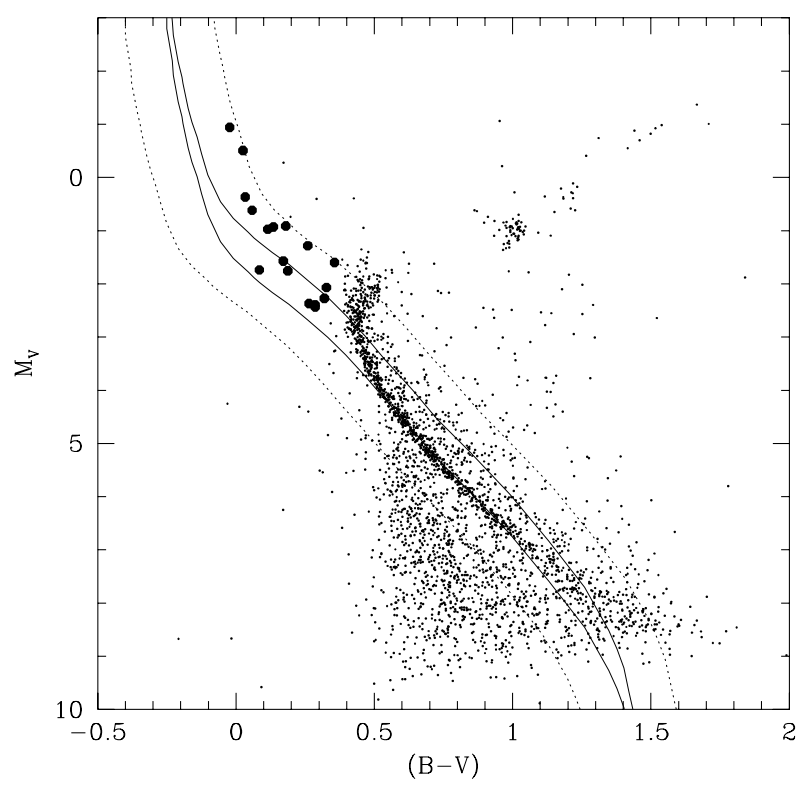

Fig. 1. Colour-magnitude diagram of the open cluster NGC 7789. Solid lines: theoretical ZAMS for single stars and for binaries with equalmass components. Dashed lines: same sequences shifted by 0.15 mag in colour and magnitude to take photometric errors and differential reddening into account. Filled circles: BSS candidates.

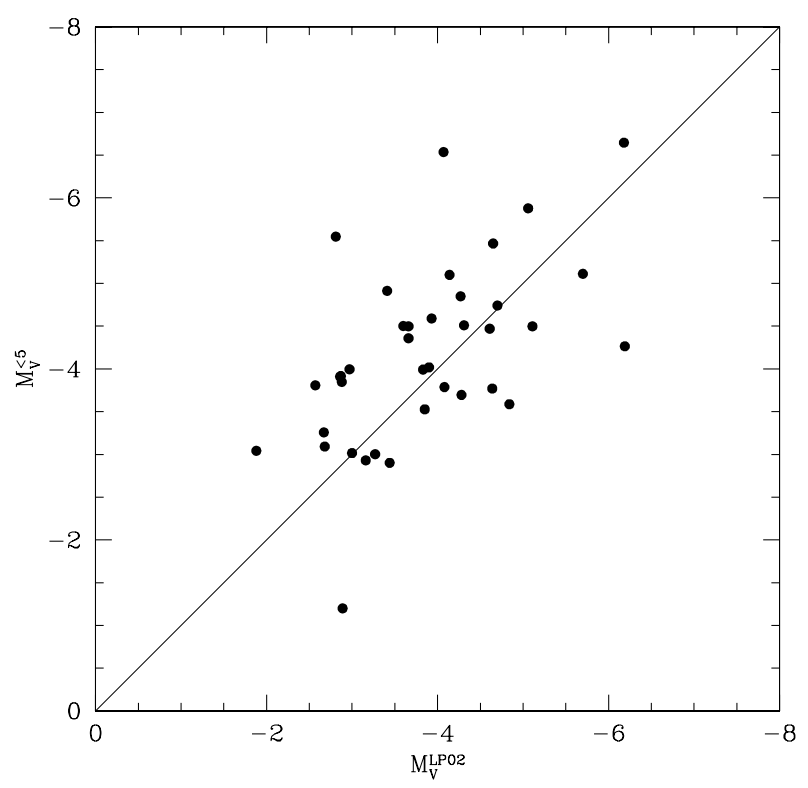

Fig. 2. Comparison between our integrated luminosity estimates and those published by LP02. The solid line merely shows the relation $M_{V}^{<5}=M_{V}^{L P 02}$.

clusters. Thirty-eight out of 59 clusters in our sample were listed in their catalogue. Figure 2 shows the comparison of LP02 values with respect to our values for the 38 clusters in common. There is overall agreement, although our integrated magnitudes are systematically slightly brighter, probably due to contamination.

\subsection{Observed number of BSS}

The upper and lower panels of Fig. 3 show the trend in the observed number of BSS stars $\left(N_{\mathrm{BSS}}\right)$ in each cluster vs. $M_{V}^{<5}$ and $M_{V}^{\mathrm{LP} 02}$, respectively. This figure is an extension to
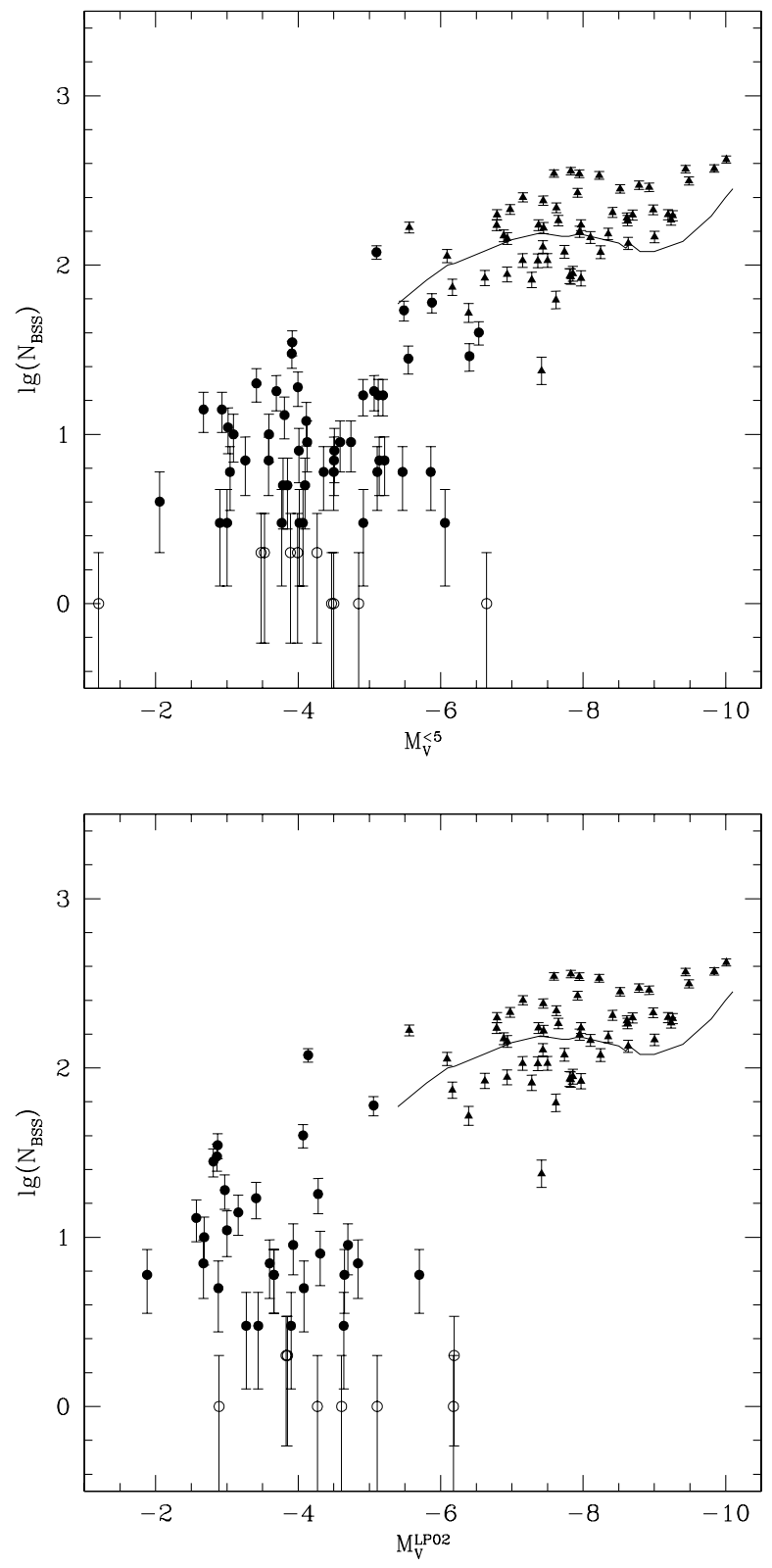

Fig. 3. Correlation between $\log N_{\mathrm{BSS}}$ and the integrated magnitude. Circles represent OC data, open symbols are used to mark clusters were only 1 or 2 BSS candidate were found. The GC data from PDK04 are plotted using triangles. The solid line shows the theoretical prediction by Davies et al. (2004).

lower luminosity and into the OC mass regime of Fig. 1 in Davies et al. (2004). The number of BSS is generally less in OCs than in GCs. Though with larger dispersion, the number of OC BSS versus total magnitude seems to lie on the prolongation of the trend indicated by the lower-luminosity GCs and by the models developed by Davies et al. (2004) (Fig. 3). We would like to stress here that the model developed by Davies et al. (2004) is not applicable to the OC environment. It is shown here only as a useful comparison. The larger dispersion of the OC data with respect to the GC ones is due to the spread in age. Older clusters have a larger number of BSS. This effect could not be observed in the GC case, as the spread in age is much less significative. The effects of age on the BSS population in OCs will be discussed further in the next sections. 


\subsection{Specific BSS frequencies}

In general, we can expect that more massive clusters contain, on average, more stars in any evolutionary branch, including BSS. Therefore, to properly study the BSS properties in different clusters, it is common practice to normalise the number of BSS to some global cluster parameter or to the total number of stars in some well-defined region of the CMD.

We considered two different normalisations in order to estimate the relative frequency of BSS in each cluster:

- $N_{\mathrm{BSS}} / \mathcal{M}_{\mathrm{tot}}$ : number of BSS normalised to the total mass (expressed in solar masses $\mathcal{M}_{\odot}$ ), derived from the total luminosity, assuming $\mathcal{M}_{\text {tot }} / \mathcal{L}_{\text {tot }}=1.5 \mathcal{M}_{\odot} / \mathcal{L}_{\odot}$;

- $N_{\mathrm{BSS}} / N_{\mathrm{cl}}$ : number of BSS normalised to the number of clump stars (i.e., stars on the horizontal branch, when this feature was clearly identified in the CMD).

In the following, we discuss the relevant correlations that were found between the derived BSS frequency and other cluster parameters.

\section{- Normalisation with total mass}

Figure 4 shows the logarithm of the specific frequency derived by normalising the total number of BSS to the cluster total mass $\left(N_{\mathrm{BSS}} / \mathcal{M}_{\mathrm{tot}}\right)$ plotted as a function of the logarithm of age. There is a clear correlation between these two quantities: older clusters have a larger relative number of BSS. A marginal correlation between these two quantities has already been found by AL95; however, we believe that this is now much more evident with the new catalogue.

PDK04 show that the relative number of BSS in GCs anticorrelates with the cluster total luminosity, in the sense that less luminous (less massive) GCs have a larger relative number of BSS. Recently, this anticorrelation has been further extended to very low-luminosity GCs by Sandquist (2005). Figure 5 shows the specific frequency of BSS vs. the integrated magnitude. The anticorrelation with the total magnitude continues down to $M_{V}^{<5}<-5$ : less massive clusters have, on average, a higher BSS frequency. In the smallest clusters of our sample, the BSS relative frequency is about two orders of magnitude higher than in the most massive GCs of PDK04.

\section{- Normalisation with clump stars}

The selection of clump stars in our CMDs was often very uncertain. The clump region in OCs is much less clearly defined and much less populated than the horizontal branch region in GCs. Only 31 out of the 59 clusters show a clear clump population. These are the oldest clusters in our sample (all older than $\sim 10^{9}$ years). The number of clump stars in every single cluster of our database is always smaller than 10 . Nevertheless we do not expect this number to be contaminated by field stars, given the very small area occupied by clump stars in OCs.

We then divided the subsample of 31 clusters into two groups, one containing 17 clusters where the selection of clump stars was straightforward and reliable, and another containing 14 clusters where the selection was uncertain mainly due to the small number of clump stars.

Figure 6 shows the trend of $\log \left(N_{\mathrm{BSS}} / N_{\mathrm{cl}}\right)$ vs. $M_{V}^{<5}$, and the GC data from PDK04, who calculated the BSS frequency by normalising the total number of BSS to the total number of horizontal branch stars, which is analogous to our normalisation to the clump stars. Noteworthy, and even more clearly so than in Fig. 3, the trend in relative number of BSS vs. integrated magnitude already seen for the globular clusters also

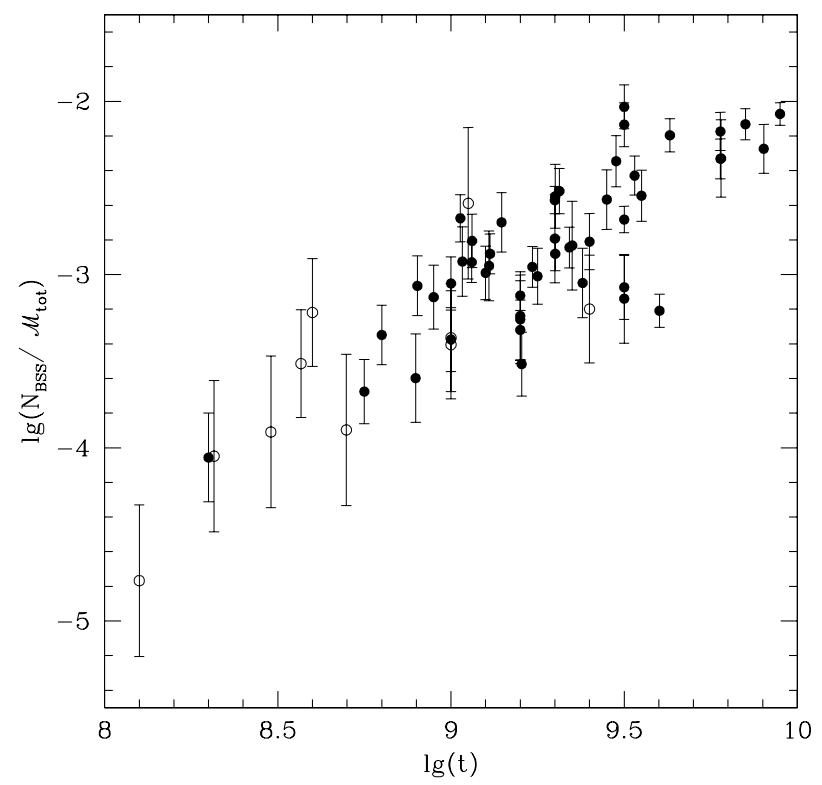

Fig. 4. The figure shows the trend in $\log \left(N_{\mathrm{BSS}} / \mathcal{M}_{\mathrm{tot}}\right)$ vs. the logarithm of age. Open symbols are used to mark clusters with only 1 or 2 BSS candidates.

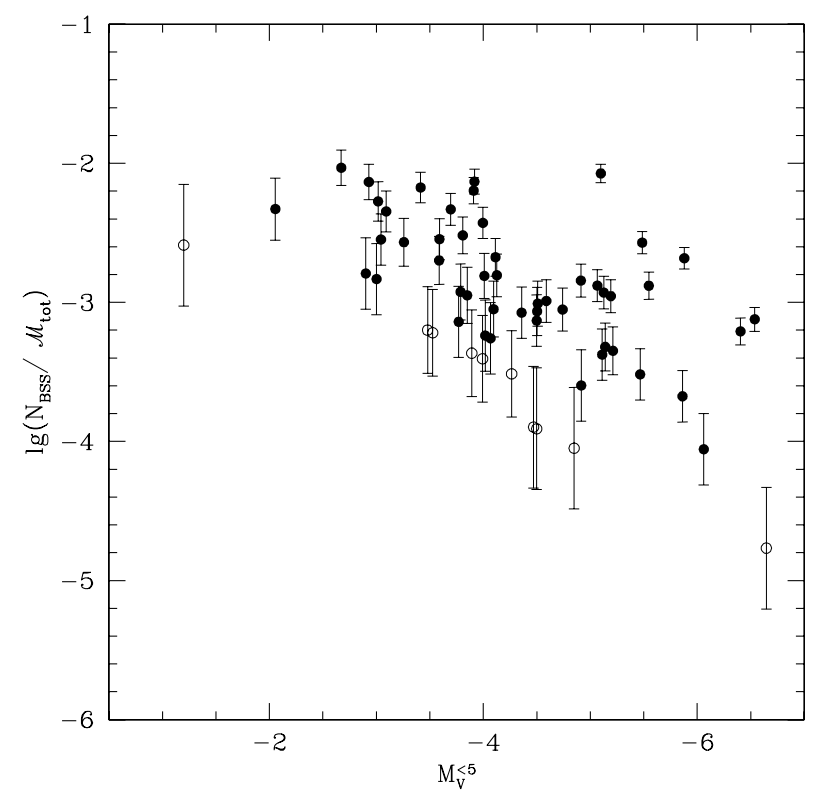

Fig. 5. The figure shows the trend in $\log \left(N_{\mathrm{BSS}} / \mathcal{M}_{\mathrm{tot}}\right)$ vs. the integrated magnitude. Open symbols are used to mark clusters with only 1 or 2 BSS candidates.

extends to the OCs. Finally, Preston \& Sneden (2000) have estimated a ratio $N_{\mathrm{BSS}} / N_{\mathrm{HB}} \sim 4$ among field stars that agrees with the extrapolation of the correlation in Fig. 6 at fainter integrated magnitudes.

\section{Modelling the expected number of BSS in OCs}

Davies et al. (2004) interpret the anticorrelation between the frequency of BSS and the total cluster mass in terms of the evolution of primordial binaries, which is affected by the stellar encounters. However, the previous section also shows a clear correlation between the number of BSS normalised to the total mass of the cluster and the age of the cluster itself, with younger 


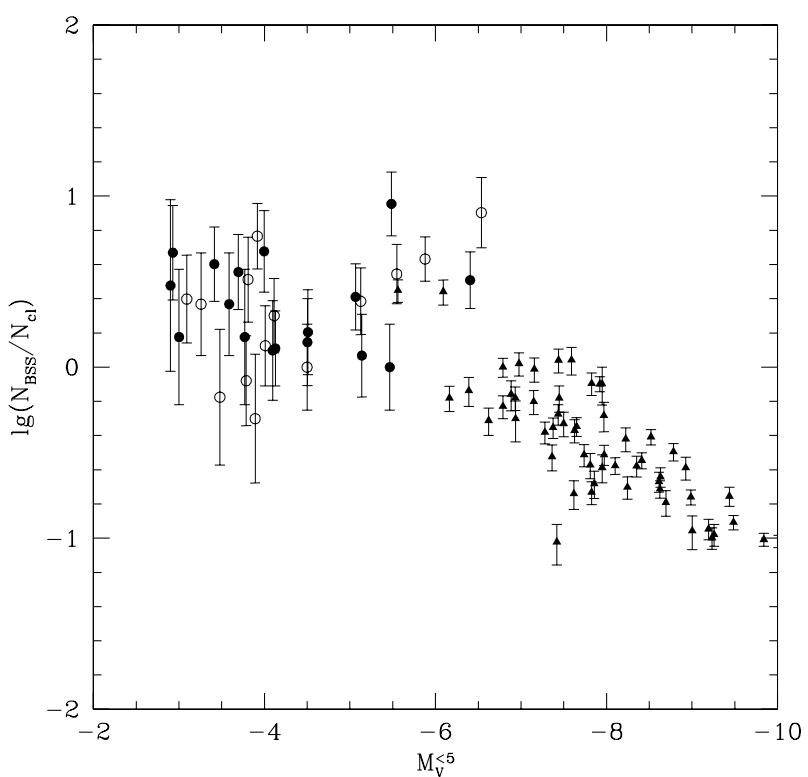

Fig. 6. The figure shows the trend in $\log \left(N_{\mathrm{BSS}} / N_{\mathrm{cl}}\right)$ vs. the integrated magnitude. Circles represent $\mathrm{OC}$ data and open symbols have been used to mark clusters where the selection of clump stars was particularly uncertain. The GC data from PDK04 are plotted using triangles.

clusters having a lower relative number of BSS with respect to the older ones. The GCs in the PDK04 catalogue were all coeval, with ages ranging from 8 to $11 \mathrm{Gyr}$ (De Angeli et al. 2005). The OCs have a much wider spread in age, from $10^{8}$ to $10^{10} \mathrm{yr}$. This property offers some insight into the possible connection between cluster evolution and BSS formation.

\subsection{Model description}

In order to further investigate this result and in an attempt to interpret it, we have developed a model following some simple prescriptions. We first assume that in OCs the number of BSS formed through direct collision (dynamical BSS) is negligible compared to the number of those formed through the evolution of primordial binaries (primordial BSS). This assumption is reasonable when considering the following facts:

- In the first instance, one could consider the estimated number of encounters involving single stars. The time-scale for a single star to undergo an encounter with another star may be approximated by (Binney \& Tremaine 1987)

$\tau_{\text {enc }} \approx 7 \times 10^{10} \mathrm{yr}\left(\frac{10^{5} \mathrm{pc}^{-3}}{n}\right)\left(\frac{V_{\infty}}{10 \mathrm{~km} \mathrm{~s}^{-1}}\right)\left(\frac{R_{\odot}}{R_{\min }}\right)\left(\frac{\mathcal{M}_{\odot}}{\mathcal{M}}\right)$,

where $n$ is the typical number density (calculated as the total number of stars divided by the volume in $\mathrm{pc}^{3}$ ), and $\mathcal{M}$ the typical combined mass of the two colliding stars (assumed to be of the order of $2 \div 3 \mathcal{M}_{\odot}$ ). The value of $V_{\infty}$ can be calculated for each cluster as $\sqrt{2 G \mathcal{M}_{\text {cluster }} / R_{\text {cluster }}}$. The ratio between the age of each cluster $\left(\tau_{\mathrm{cl}}\right)$ and $\tau_{\text {enc }}$

$N_{\mathrm{enc}}=\frac{\tau_{\mathrm{cl}}}{\tau_{\mathrm{enc}}}$

should give a reasonable estimate of the number of encounters that could produce BSS. The ratio $N_{\text {enc }} / \mathcal{M}_{\text {tot }}$ is negligible compared to the number of observed BSS (the expected number being between 1 and 2 orders of magnitude lower than the observed number of BSS). Moreover, this is likely to overestimate the number of collisional BSS because 1) not all the dynamical interactions will produce BSS, 2) only BSS formed in the past $\approx 1$ Gyr (approximate estimate of the BSS lifetime) will be visible today. On the other hand, this estimate does not take dynamical interactions involving binaries into account. These are expected to be more frequent due to the larger cross section. However, even in this case, we do not expect all the encounters to produce BSS.

- The fraction of binaries undergoing a dynamical interaction can be estimated using the former formula but adopting a value for $R_{\min }$ typical of the size of a binary. This will obviously depend on the properties of the primordial binary population, and will be higher for low-velocity dispersion clusters (as the velocity dispersion decreases, wider binaries qualify as "hard"). Observational evidence (for a discussion see Sandquist 2005 ) shows that at least $\approx 25 \%$ of the BSS in M 67 are dynamically formed. However, assuming a value of the order of a $100 \mathrm{AU}$ for $R_{\min }$ and using a realistic estimate of the cluster velocity dispersion in Eq. (1), one finds that the mean expected fraction of binaries experiencing dynamical interactions for the clusters in our sample is more than 10 times lower than the one in M 67. Thus we expect the fraction of dynamical BSS in most of our clusters to be of the order of a few percent, assuming an equal binary fraction for all clusters. More careful considerations should also take the age of the clusters into account. Our sample spans an age range between $10^{8}$ and $10^{10} \mathrm{yr}$, and it is not at all clear how age influences the collision rate. The widest binaries (those with higher cross-section) are likely to interact earlier on, and their BSS products might not be visible today.

Our simple model builds up a cluster with both a single star and a binary population. All the components are drawn randomly from the same IMF (we adopted the IMF of Eggleton et al. 1989). The masses are generated using the equation

$\mathcal{M}=\frac{0.19 x}{(1-x)^{0.75}+0.032(1-x)^{0.25}} \mathcal{M}_{\odot}$

with a series of random numbers $x$. The binary fraction is assumed to be of the order of 0.5 .

Binary systems will form BSS only if one of the two following criteria is verified:

1. either the mass ratio between the secondary and the primary $\left(q=\mathcal{M}_{2} / \mathcal{M}_{1}\right)$ is higher than a certain $q_{\mathrm{MT}}$ (and obviously lower than 1); in this case the mass transfer between the primary and the secondary is expected to be stable and to lead to the formation of a blue straggler star with an evolved companion;

2. or the mass ratio between the secondary and the primary is lower than $q_{\mathrm{MT}}$ but higher than a certain $q_{\mathrm{CE}}$, in which case the mass transfer will probably be unstable and lead to a merger of the two components of the binary system, this time leading to the formation of a single blue straggler star.

If $q<q_{\mathrm{CE}}$, we believe that a common envelope will form and no BSS will originate from such systems.

The choice of the values for $q_{\mathrm{MT}}$ and $q_{\mathrm{CE}}$ was taken in consideration of the following facts. Conservative mass transfer from a more-massive donor to a less-massive receiving star is often unstable (Frank et al. 2002). However, if the mass ratio is close to unity, mass transfer will reverse the mass ratio (i.e. the donor will quickly become the less-massive star) and mass transfer may proceed in a stable fashion. There is evidence that 
this must be the case for at least some BSS, since some field BSS have been found in wide binaries presumably with white dwarfs (Preston \& Sneden 2000). As a reasonable example here, we take this critical mass ratio to be $q_{\mathrm{MT}}=0.85$.

In systems with a mass ratio below this value, mass transfer will be unstable, resulting in the merger of the two stars and the formation of a blue straggler, if the mass exceeds the current turnoff. In systems with a very low mass-ratio, the less-massive star will be much denser than the primary. Rather than merge, the two stars will form a common envelope system where gas from the more-massive (and less dense) envelope will smother the less-massive (and denser) star, forming a common envelope around it and the core of the more-massive star. In this second case, the common envelope will be ejected as the core and the low-mass star spiral together, and no blue straggler will be formed. Here we assume this occurs for $q<0.4$ (i.e. $q_{\mathrm{CE}}=0.4$ ).

For each binary, leading to a blue straggler, three different times are defined:

1. $t_{\mathrm{ON}}$ : the time at which the primary evolves off the main sequence, and thus presumably starts the mass transfer on the secondary or the merger between the two companions. The main sequence lifetimes were calculated by applying the analytic formulae for stellar evolution as a function of mass and metallicity given by Hurley et al. (2000), assuming a typical metallicity close to the solar one.

2. $t_{\mathrm{BSS}}$ : the lifetime of the formed star. This is calculated in a different way depending on the formation process: (1) for mass transfers, this is the main sequence lifetime $(\tau)$ of a star of mass $\mathcal{M}_{2}+5 / 6 \mathcal{M}_{1}$ as the expected transferred mass is about $5 / 6$ of the mass of the primary; (2) for mergers, this will have to be calculated considering the fraction of the core mass left available in the two merging stars. In particular, if $\mathcal{M}_{1}$ and $\mathcal{M}_{2}$ are the starting total masses of the two components, then at the time $\tau_{1}$ when the primary evolves off the main sequence, we will have that the core mass of the primary

$\mathcal{M}_{\text {core }, 1}=a \mathcal{M}_{1}^{b}$,

(where $a=0.125$ and $b=1.4$, van den Heuvel 1994) has already been transformed into helium $\left(\mathcal{M}_{\mathrm{core}, 1}=\mathcal{M}_{\mathrm{He}, 1}\right)$. At the same time, assuming that the hydrogen burning proceeds linearly with time during the evolution on the main sequence, the secondary will have burned

$\mathcal{M}_{\mathrm{He}, 2}=a \mathcal{M}_{2}^{b} \tau_{1} / \tau_{2}=\mathcal{M}_{\text {core }, 2} \tau_{1} / \tau_{2}$.

The core mass of the merger product will be $\mathcal{M}_{\text {core, } 1+2}=$ $a\left(\mathcal{M}_{1}+\mathcal{M}_{2}\right)^{b}$, but not all of it will be hydrogen. In fact the hydrogen core mass will be $\mathcal{M}_{\mathrm{H}, 1+2}=\mathcal{M}_{\text {core, } 1+2}-$ $\mathcal{M}_{\mathrm{He}, 1}-\mathcal{M}_{\mathrm{He}, 2}$. The main sequence lifetime will then be $\tau_{1+2} \mathcal{M}_{\mathrm{H}, 1+2} / \mathcal{M}_{\text {core }, 1+2}$.

3. $t_{\mathrm{OFF}}=t_{\mathrm{ON}}+t_{\mathrm{BSS}}$ : the expected time at which the formed star evolves off the main sequence, leaving the CMD area where BSS are selected.

Finally, the model loops over all the time range of interest $\left(10^{8}<t<10^{10} \mathrm{Gyr}\right)$ and for each time (i.e. age) counts all the BSS having $t_{\mathrm{ON}}<t<t_{\mathrm{OFF}}$. At each time $t$, the total mass of the cluster is estimated as the sum of all the unevolved single stars and binaries (we consider only those binaries in which the primary has not yet evolved off the main sequence, $\tau_{1}<t$ ). The total mass will then be used to normalise the total number of BSS to allow a comparison with the observed frequencies.

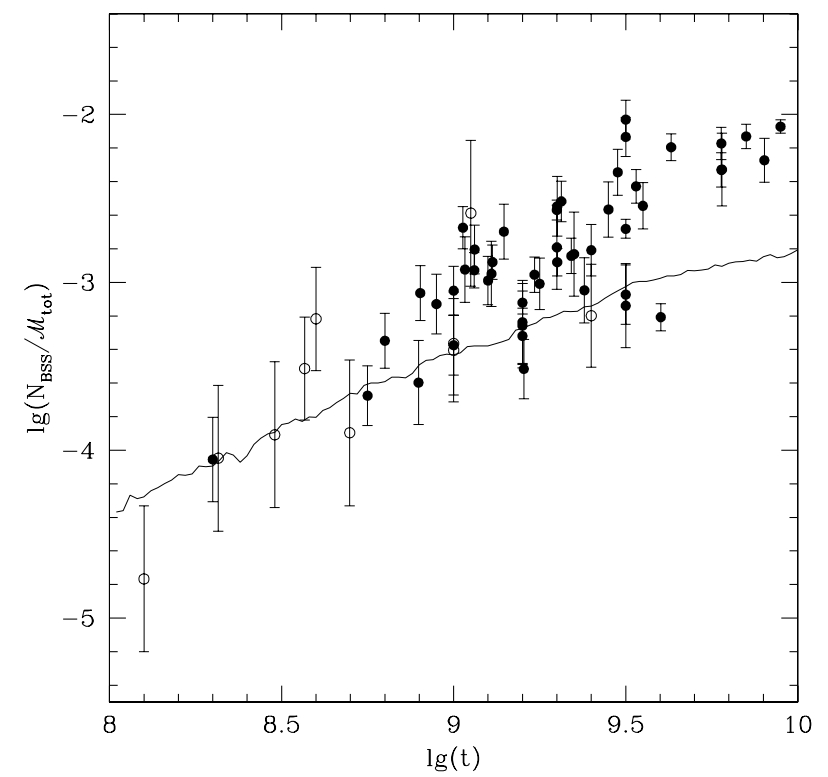

Fig. 7. Comparison between expected $\log \left(N_{\mathrm{BSS}} / \mathcal{M}_{\mathrm{tot}}\right)$ and observed frequencies of BSS in OC. The solid line represents the result of our model. Observed data are shown with filled and open circles, open circles marking clusters with only 1 or 2 BSS candidates.

\subsection{Interpreting the empirical results}

The resulting expected time evolution of the relative number of BSS normalised to the total mass is only slightly dependent on the assumed values for $q_{\mathrm{MT}}$ and $q_{\mathrm{CE}}$. Changing the values of these parameters shifts the expected trend toward higher or lower frequencies but does not influence the evolution rate significantly. Clearly many uncertainties must be taken into account when proceeding with this kind of analysis. Observationally, the uncertainty on the mass-luminosity ratio and thus on the total mass clearly affects the level of the trend. Theoretically, the adopted values for the parameters $q_{\mathrm{MT}}$ and $q_{\mathrm{CE}}$ can also shift the expected trend towards higher or lower frequencies, and our values, although reasonable, have not been precisely determined in previous works. For these reasons, we concentrate in the following more on the general trend of the correlation than on the level of the relative numbers of BSS. Figure 7 shows the expected trend assuming $q_{\mathrm{MT}}=0.85$ and $q_{\mathrm{CE}}=0.4$, with the corresponding observed quantities. As shown in the plot, the expected trend of BSS frequency with age is flatter than the observed one. Older OCs show a higher number of BSS per unit mass than expected.

However, one important aspect has not been taken into consideration by our model. Mass loss plays a very important role in OCs, so we should account for it when defining the total normalising mass. Since they are more massive than normal stars, BSS sink toward the centre quickly after formation, which implies that the BSS population should be less affected by mass loss than the normal stellar population. At increasing ages, OCs tend to lose more and more mass. Therefore the normalising factor becomes smaller and smaller, steepening the relation between normalised number of BSS and cluster age in Fig. 7. Mass loss also depends on the cluster's total mass, which can partly account for the wide spread of the observed quantities, together with the large uncertainties in the mass estimates.

Recently, a couple of papers have addressed the issue of mass loss in stellar clusters. Tanikawa \& Fukushige (2005, hereafter TF05) and Lamers et al. (2005, hereafter L05) present Nbody simulation results, predicting mass loss timescales of star 


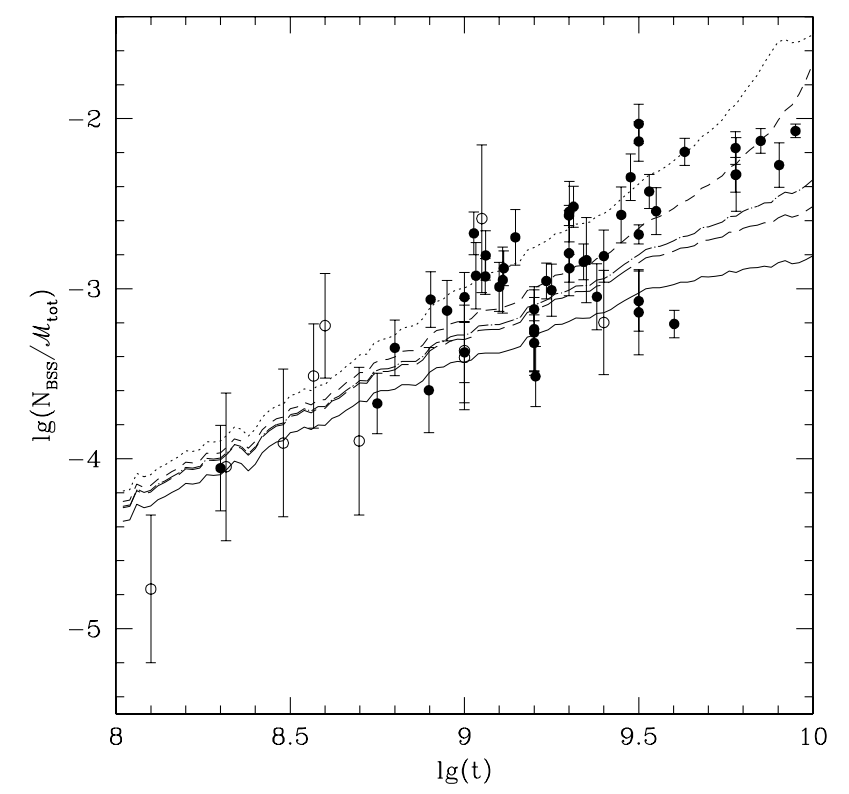

Fig. 8. Comparison between expected $\log \left(N_{\mathrm{BSS}} / \mathcal{M}_{\mathrm{tot}}\right)$ and observed frequencies of BSS in OC. The solid line represents the result of our model. Observed data are shown with filled and open circles, open circles marking clusters with only 1 or 2 BSS candidates. Dotted, dashed, dot-dashed and long-dashed curves reproduce respectively the expected values obtained taking into account mass loss following the results of Tanikawa \& Fukushige (2005) with $2 \mathrm{k}, 8 \mathrm{k}, 32 \mathrm{k}$, and $131 \mathrm{k}$ Nbody models.

clusters. We applied the results of both works to investigating the effect of mass loss on our predictions.

Figure 2 in TF05 reproduces the mass loss as a function of time. The curves from the first panel at the top and on the left are the most appropriate for our analysis, having lower initial concentration and higher resolution. Time was converted from Nbody units to Myr by adopting the typical cluster properties in 5 different age intervals. This gave us the typical mass loss in each interval, which we then applied to the results of our model, assuming that BSS are not affected by mass loss. We repeated this procedure for all the curves in the first panel of Fig. 2 of TF05 (2k, 8k, 32k, 131k, where k stands for 1000 particles), and Fig. 8 shows the different results. The estimates obtained using the $2 \mathrm{k}$ and $8 \mathrm{k}$ Nbody models are probably the more realistic ones, all our clusters having less than 2100 stars.

The observed points nicely occupy the region between the lines, representing the two most extreme scenarios (no mass loss, and large mass loss affecting only normal stars), with only a few points falling outside this region. This may suggest that BSS are indeed affected by mass loss as well, although not as much as normal stars. This preliminary analysis confirms that our simple model with approximated mass-loss estimates can reproduce the distribution of the observed points quite well.

To confirm these conclusions, we tried to estimate the mass loss with a different approach. L05 provide a set of analytical formulae to estimate the mass loss due to both stellar evolution and tidal effects. Stellar evolution is taken into account by our model. Due to the analytical form of the recipe provided by L05, it is convenient to eliminate the tidal effects from the observed data instead of adding them to the expected quantities. For this reason, we use Eq. (7) of the L05 paper to estimate the initial mass of the clusters in our catalogue starting from the present observed mass (note that this equation takes into account the mass loss due to both stellar evolution and tidal effects). Then using

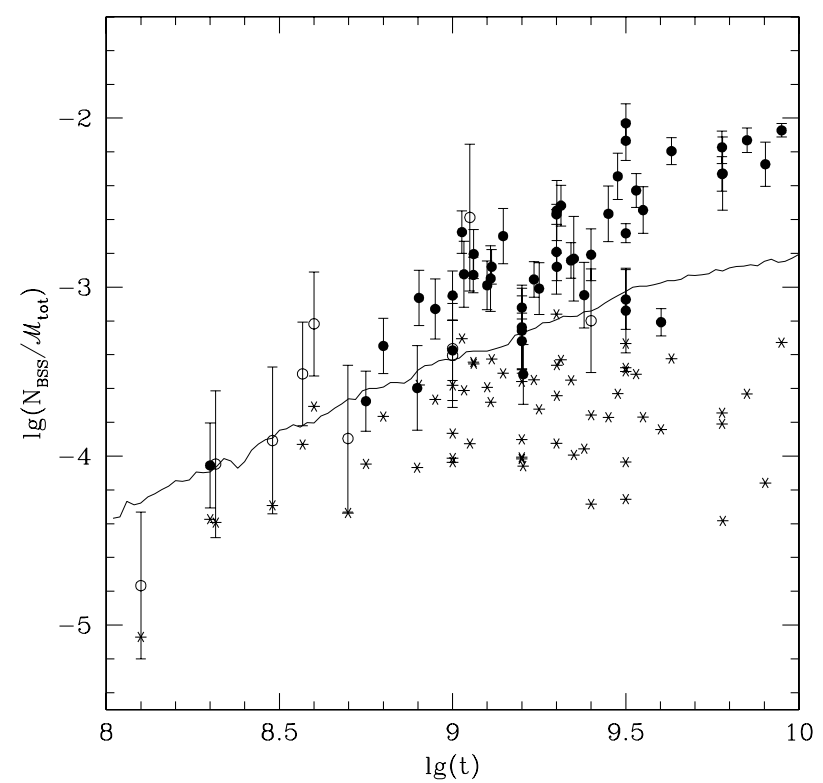

Fig. 9. Comparison between expected $\log \left(N_{\mathrm{BSS}} / \mathcal{M}_{\mathrm{tot}}\right)$ and observed frequencies of BSS in OC. The solid line represents the result of our model. Observed data are shown with filled and open circles, open circles marking clusters with only 1 or 2 BSS candidates. Asterisks are the observed BSS frequencies after correcting for mass loss due to tidal effects using the recipe suggested by Lamers et al. (2005) as explained in the text.

Eqs. (3) and (2) of L05, we correct the calculated initial mass taking the mass loss due only to stellar evolution into account. The obtained mass estimates will be affected by mass loss due to stellar evolution but not by mass loss due to tidal effects, i.e. will be comparable with the masses calculated in our model (which does not account for tidal effects but includes stellar evolution).

Figure 9 shows the observed values, the corresponding values corrected for mass loss due to tidal effects applied to normal stars only and our model predictions. The corrected values represent the case where the mass loss is the most evident. If BSS were also affected by mass loss, then the observed frequency would lie between the observed values and the corrected ones. The trend predicted by the model falls in between the observed quantities and those corrected for the maximum tidal effect. Clearly the results obtained with both approaches are consistent with each other thus suggesting that tidal effects play an important role in OC and that they probably affect the number of observed BSS, as well as the total mass.

In this respect we wish to make one last consideration. The fraction of binaries undergoing a dynamical interaction can be estimated using Eq. (1) as explained in Sect. 4.1. This fraction can be estimated for each cluster in our sample. For older clusters the expected values are higher than those obtained for the younger ones. All but one of our clusters have an estimate for the fraction of binaries undergoing a dynamical interaction lower than the one calculated for M 67, the mean value being about one order of magnitude lower. Remembering that at least $25 \%$ of the BSS population of M 67 appears to be dynamically formed, this could imply that several of our clusters could show a non negligible dynamical population of BSS. This would also cause the frequency of BSS in older clusters to be higher than the predictions of our model.

Finally, the normalisation with the total mass adopted for the observed number of BSS, is sensitive to the choice of the mass-luminosity ratio. The dependence of $\mathcal{M}_{\text {tot }} / \mathcal{L}_{\text {tot }}$ vs. age is affected by both evolutionary and dynamical effects. Both stellar 
evolution and mass loss due to tidal interactions are expected to cause the mass-luminosity ratio in old clusters to be higher than in young clusters. This would thus bring the observed trend in better agreement with the results of our model.

As already noted in the previous section, there is a clear continuity between open and globular clusters. This is clearly shown in Fig. 3. Davies et al. (2004) use a model similar to the one implemented here to predict the trend of the total number of BSS with respect to the total magnitude.

Although in this paper we concentrate on the evolution of the BSS population with cluster age, we have also checked that the present model is consistent with the predictions of Davies et al. (2004) at the low mass limit of our OCs. At ages of the order of the Galactic GC ages ( $\approx 10 \mathrm{Gyr})$, the present model predicts $\log N_{\text {BSS }} \simeq 3.2$. From the total mass, predicted by the model for clusters with this age, we can estimate the total magnitude assuming a mass-to-light ratio of the order of 3, as in Davies et al. (2004). We obtain $M_{V} \approx-9$. At this magnitude, their model predicts $\log N_{\mathrm{BSS}} \approx 2.1$, including the effects of both primordial binary evolution and stellar collisions. The dashed curve in Fig. 6 of Davies et al. (2004) shows the contribution to the total number of BSS from primordial system. This curve would be a straight line if there were no collisions; in fact, the decline for magnitudes brighter than -7.4 is due to the increasing $N_{\text {enc }}$. As discussed in Davies et al. (2004), the effect of the encounters is to fasten the evolution of primordial binaries: the more encounters we have the smaller the number of binaries able to evolve in a BSS after 10 Gyr or so. But this does not apply to the case of open clusters. As shown above, we do not expect encounters to play an important role in this environment, so that in this case we would expect the dashed curve to be linearly increasing with $M_{V}$, reaching $\log N_{\mathrm{BSS}} \approx 3.2$ at $M_{V} \approx-9$.

One further constraint must be satisfied by our model, i.e. the observed frequency of BSS among field stars. Preston \& Sneden (2000) estimate $s_{\mathrm{BSS}}=N_{\mathrm{BSS}} / N_{\mathrm{HB}}=4$ in the field. In order to compare our results with this value, we estimated the number of horizontal branch (HB) stars in our simulated clusters. We again adopted the analytic formulae for stellar evolution (Hurley et al. 2000) to evolve our simulated stars and to count the number of stars during the HB phase at each time step. Assuming then the much lower metallicity $(Z=0.0004)$ typical of field stars as in Preston \& Sneden (2000), we recomputed our expected frequency of BSS with respect to the number of HB stars. For ages of the order of $12 \div 13 \mathrm{Gyr}$, we obtained $s_{\mathrm{BSS}} \approx 2.5 \div 3$, slightly lower but of the same order of magnitude as the results obtained by Preston \& Sneden (2000). The difference can be due to the different model, stellar evolution prescriptions, and input quantities. Obviously, when estimating the frequency of BSS in the field, mass loss should no longer play an important role, and thus the correct estimate should be given by our model without any correction for mass loss.

Acknowledgements. F.d.M. and G.P. acknowledge support by the Italian MIUR, under the programme PRIN03. The authors wish to thank the anonymous referee for useful comments and suggestions that considerably improved the paper.

\section{References}

Ahumada, J., \& Lapasset, E. 1995, A\&AS, 109, 375

Benz, W., \& Hills, J. G. 1987, ApJ, 323, 614

Binney, J., \& Tremaine, S. 1987, Galactic dynamics (Princeton, NJ: Princeton University Press), 747

Davies, M. B., Piotto, G., \& de Angeli, F. 2004, MNRAS, 349, 129

De Angeli, F., Piotto, G., Cassisi, S., et al. 2005, AJ, 130, 116

Eggen, O. J., \& Iben, I. J. 1988, AJ, 96, 635

Eggen, O. J., \& Iben, I. J. 1989, AJ, 97, 431

Eggleton, P. P., Tout, C. A., \& Fitchett, M. J. 1989, ApJ, 347, 998

Frank, J., King, A., \& Raine, D. J. 2002, Accretion Power in Astrophysics: Third Edition, ed. J. Frank, A. King, \& D. Raine (Cambridge, UK: Cambridge University Press), 398

Girardi, L., Bressan, A., Bertelli, G., \& Chiosi, C. 2000, A\&AS, 141, 371

Hurley, J. R., Pols, O. R., \& Tout, C. A. 2000, MNRAS, 315, 543

Lamers, H. J. G. L. M., Gieles, M., Bastian, N., et al. 2005, A\&A, 441, 117

Lata, S., Pandey, A. K., Sagar, R., \& Mohan, V. 2002, A\&A, 388, 158

Lombardi, J. C., Rasio, F. A., \& Shapiro, S. L. 1996, ApJ, 468, 797

Mateo, M., Harris, H. C., Nemec, J., \& Olszewski, E. W. 1990, AJ, 100, 469

McCrea, W. H. 1964, MNRAS, 128, 147

Piotto, G., King, I. R., Djorgovski, S. G., et al. 2002, A\&A, 391, 945

Piotto, G., De Angeli, F., King, I. R., et al. 2004, ApJ, 604, L109

Preston, G. W., \& Sneden, C. 2000, AJ, 120, 1014

Sandquist, E. L. 2005, ApJ, 635, L73

Tanikawa, A., \& Fukushige, T. 2005, PASJ, 57, 155

van den Heuvel, E. P. J. 1994, in Interacting binaries, Lecture notes of the 22nd Advanced Course of the Swiss Society for Astronomy and Astrophysics (SSAA), held at Saas-Fee, Switzerland, 1992 (Berlin: Springer), ed. H. Nussbaumer, \& A. Orr, 263

Williams, I. P. 1964, Annales d'Astrophysique, 27, 198 